\title{
Tromboprofilaxis en el paciente quirúrgico COVID-19
}

\author{
Thromboprophylaxis in the COVID-19 surgical patient
}

Arturo Lorenzo B. MD. ${ }^{1}$, Oscarin Jiménez MD. ${ }^{2}$

\begin{abstract}
Clotting disorders have been one of the problems that clinicians have dealt with in the course of learning about this new disease. There is very little evidence and limited recommendations by scientific societies on the management of thromboprophylaxis in the COVID-19 surgical patient, in addition to the fact that the effects of this pathology on surgical injury are unknown. The thrombotic risk assessment for the COVID-19 surgical patient is unprecedented in this regard. Some predictors and risk factors for thrombosis have been systematically reported as prognostic markers, including elevation of D-dimer, fibrinogen, IL6, serum ferritin, prolongation of clotting times, detection of antiphospholipid antibodies and other independent factors that contribute to measure severity. of COVID-19 such as lactate dehydrogenase and marked lymphopenia. The foregoing predictors and risk factors for thrombosis for COVID-19 disease have been adapted adjusted to the categories present on the Caprini scale in order of increasing complexity and in relation to morbidity and mortality. In this sense, the choice of the measure or measures that each patient requires will depend on their particular risk of developing venous thromboembolic disease against the anticipated risk of surgical bleeding complications from the surgery to which they will be subjected, allowing the identification of patients likely to develop complications. through the modified Caprini scoring system and you see guide and establish optimal thromboprophylaxis strategies.
\end{abstract}

\section{Key words:}

Covid-19,

surgery,

perioperative, thromboprophylaxis

Especialista en Anestesiología. Médico Adjunto al Servicio de Anestesiología del Hospital General Dr. Luis Razetti. Barinas, Venezuela.

2 Médico Adjunto al Servicio de Anestesiología del Hospital Materno Infantil Dr. Samuel Darío Maldonado. Barinas, Venezuela.

Fecha de recepción: 02 de octubre de 2020

Fecha de aceptación: 14 de octubre de 2020

\section{ORCID}

https://orcid.org/0000-0003-3302-0537

Fuente de financiamiento:

Los autores declaran no haber recibido ningún tipo de financiamiento al realizar el presente estudio.

Correspondencia:

Arturo Lorenzo Betancourt.

arturolorenzo123@gmail.com 


\section{RESUMEN}

Los trastornos de la coagulación han sido uno de los problemas con que los clínicos han lidiado en el transcurso del conocimiento de esta nueva patología. Existe muy escasa evidencia y limitadas recomendaciones por parte de las Sociedades Científicas sobre el manejo de la tromboprofilaxis en el paciente quirúrgico COVID-19, aunado a que se desconoce los efectos de esta patología sobre la injuria quirúrgica. La evaluación del riesgo trombótico en cuanto al paciente quirúrgico COVID-19 no cuenta con precedentes al respecto. Algunos predictores y factores de riesgo de trombosis se han informado sistemáticamente como marcadores pronóstico, entre ellos la elevación del dímero-D, fibrinógeno, IL6, ferritina sérica, prolongación de tiempos de coagulación, detección anticuerpos antifosfolípidos y otros factores independientes que contribuyen a medir la gravedad de COVID-19 como lo es la lactato deshidrogenasa y la linfopenia marcada. Los anteriores predictores y factores de riesgo de trombosis para la enfermedad de COVID-19 se han adaptado ajustados a las categorías presentes en la escala de Caprini en orden creciente de complejidad y en relación a la morbimortalidad. En este sentido la elección de la o las medidas que requiere cada paciente dependerá de su riesgo particular de desarrollar enfermedad tromboembólica venosa contra el riesgo anticipado de complicaciones hemorrágicas quirúrgicas de la cirugía a la que será sometido, permitiendo identificar a los pacientes susceptibles de desarrollar complicaciones a través del sistema de puntuación modificado de Caprini y a la vez guiar y establecer estrategias óptimas de tromboprofilaxis.

\section{Palabras clave:}

COVID-19,

cirugía,

perioperatorio, tromboprofilaxis

\section{Introducción}

D esde los primeros casos publicados de la infección por este nuevo coronavirus denominado SARS-CoV-2[1], la coagulopatía ha sido uno de los problemas con que los clínicos han lidiado en el transcurso del conocimiento de esta nueva patología[2], el SARS-CoV-2 inicia la infección celular al unirse al receptor de la enzima convertidora de angiotensina 2 (ACE2) el cual se expresa ampliamente en los tejidos humanos, incluyendo el epitelio alveolar, corazón, hígado, riñón y las células endoteliales[3], por consiguiente, el tejido vascular es bastante susceptible, ya que expresa altamente la enzima convertidora de angiotensina-2 (ACE-2) y se ha demostrado que el virus SARS-CoV-2 infecta a los vasos sanguíneos e induce daño vascular in vitro e in vivo, relacionándose esta lesión endotelial con el origen de la coagulopatía y la activación de la hemostasia que conducen a trastornos trombóticos observados en pacientes con COVID-19[4].

Con frecuencia la enfermedad por COVID-19 induce hipercoagulabilidad con un grado variable de inflamación que aumenta los niveles de factores de coagulación procoagulantes e interrumpe la homeostasis normal de las células endoteliales vasculares, describiéndose dos procesos patológicos de la coagulación tanto a nivel microcirculatorio donde existe una lesión local directa vascular y endotelial que produce formación de coágulos microvasculares y angiopatía revelados en los estudios post mortem del pulmón con infiltración mononuclear y polimorfonuclear junto con apoptosis de células endoteliales y mononucleares, así como en la circulación sistémica, debido a la hipercoagulabilidad con hiperfibrinogenemia, lo que resulta en microangiopatía, formación de trombos locales y en un defecto de coagulación sistémica que conduce a la trombosis de grandes vasos y complicaciones tromboembólicas importantes[5],[6]. Cui et al.[7]. Examinaron la trombosis en los miembros inferiores de pacientes no sintomáticos con neumonía por COVID-19 tratados en UCI mediante ultrasonografía encontrando una prevalencia del 25\% de los casos, sin embargo, los hallazgos de trombosis vascular pulmonar por microangiopatía trombótica o embolia pulmonar se informan hasta en el $40 \%$ de los pacientes hospitalizados con COVID-19[8],[9].

La combinación de la fisiología observada y la evidencia patológica emergente apunta hacia un proceso de enfermedad vascular como factor contribu- 
yente en la patogénesis de COVID-19[10]. Por lo que, la siguiente revisión tiene como objetivo orientar las pautas del manejo de tromboprofilaxis en el paciente quirúrgico COVID-19 según las recomendaciones basadas en la evidencia de esta patología emergente.

\section{Infección por SARS-CoV-2/COVID-19 cirugía y tromboprofilaxis}

Existe muy escasa evidencia y limitadas recomendaciones en el momento actual por parte de las Sociedades científicas sobre el manejo de la tromboprofilaxis en el paciente quirúrgico COVID-19[11],[12] y aún más sobre de qué manera afecta directamente la cirugía al paciente con esta patología. Sin embargo, el estrés quirúrgico se asocia con un cambio exponencial en el perfil inmune, especialmente en los pacientes sometidos a cirugía mayor, particularmente, cirugía cardíaca, torácica o abdominal; estos presentan niveles elevados de citocinas proinflamatorias como IL-6 la cual está asociadas con complicaciones postoperatorias, con un odds-ratio de hasta 56,4[13]. Todos estos elementos nos permiten suponer que el hecho de agregar estrés quirúrgico a un paciente con COVID-19 el cual ya por su estado fisiopatológico protrombótico puede ser perjudicial en pacientes sometidos a procedimientos quirúrgicos y en especial a la cirugía mayor, por lo que, se ha descrito un aumento de la morbilidad y mortalidad en los pacientes intervenidos quirúrgicamente por grupos europeos y asiáticos[14],[15],[16].

En este contexto de estallido de inflamación, inmovilización, coagulación activada y fibrinólisis suprimida, el riesgo de enfermedad tromboembólica venosa TEV y embolia pulmonar EP se encuentra aumentado al quebrantar por completo la tríada de Virchow[5]. La infección por COVID-19 se asocia con una coagulopatía caracterizada por un aumento de los factores procoagulantes como el fibrinógeno, junto con un fuerte aumento del dímeros-D el cual se ha relacionado con una mayor tasa de mortalidad[2],[17], además, en términos de señal protrombótica se han asociado la presencia de anticuerpos antifosfolípidos representados por anticardiolipina $(\mathrm{aCL})$ y anti- $\beta 2$ glucoproteína I (aß2GPI) 2 y de anticoagulante de lupus (LA) en estos pacientes, lo que agrava aún más el estado protrombótico de base de la enfermedad[4]. Todos estos perfiles procoagulantes en pacientes con COVID-19 alentaron a la Sociedad Internacional de Trombosis y Hemostasia a proponer anticoagulación preventiva en pacientes hospitalizados con COVID-19 mas no se tiene recomendaciones en el manejo de pacientes quirúrgicos positivos[18].
La trombosis venosa profunda (TVP) y la embolia pulmonar (EP) (colectivamente, TEV) son complicaciones bien reconocidas, clínicamente importantes y potencialmente devastadoras que pueden ocurrir después de procedimientos quirúrgicos mayores, definidos como cualquier intervención quirúrgica que conlleve un factor de riesgo mayor de trombosis al mínimo procedimiento realizado en quirófano que requiera de formación especializada. La importancia de las medidas preventivas para minimizar el riesgo de TEV después de una cirugía mayor se ha reconocido durante décadas; sin embargo, incluso con el uso de profilaxis, la cirugía en el paciente no COVID-19 representa aproximadamente el $25 \%$ de los TEV observados[19].

\section{Factores de riesgo trombótico y de gravedad asociados a COVID-19}

Los factores de riesgo de trombosis y predictores de mortalidad asociado con la infección por COVID-19 se encuentran ampliamente descritos[21],[22],[23], [24],[25]. Entre las alteraciones de la coagulación con mayor valor pronóstico encontramos que uno de los primeros y más comunes hallazgos de laboratorio observados en pacientes con COVID-19 que requieren hospitalización fue la marcada elevación del dímero-D. Un dímero-D alto es inespecífico y a menudo se asocia con diversas afecciones médicas, como infecciones, traumatismos o incluso hospitalización; sin embargo, en el contexto de la infección por COVID-19, se ha informado sistemáticamente como un marcador de pronóstico deficiente que se asocia con un curso crítico y una mayor mortalidad[2],[26],[27],[28],[29],[30]. Un punto de corte de $0,5 \mathrm{mg} / \mathrm{L}$ es más frecuente en pacientes con enfermedad grave que en aquellos sin ella con (60\% vs 43\%, $p=0,002)$ [27]. Así mismo, el aumento considerable de los niveles de fibrinógeno por la supresión fibrinolítica con un promedio visto por tromboelastometría 7,8 g/L, y aumento de la fuerza del coágulo son perfiles procoagulantes observados en los pacientes graves[34]. En cuanto al tiempo de protrombina TP, Zhou y Yu[31] informaron un tiempo de protrombina significativamente mayor (> $16 \mathrm{~s})$ en los no sobrevivientes $(n=54)$ en comparación con los sobrevivientes $(n=137)(13 \%$ vs $3 \% ; p=$ 0,0004 ) otros estudios han encontrado patrones similares en los pacientes no sobrevivientes[2]. Respecto al recuento de plaquetas los estudios difieren en muy pocos cambios entre los pacientes graves y las formas leves de la enfermedad[27],[28],[29], sin embargo, un análisis reciente informó una mortalidad hospitalaria de $92,1 \%$ para aquellos con un recuento de plaque- 
tas entre 0 y 50 riesgo relativo 13,68 (IC del 95\%: 9,89-18,92)[32]. La detección Anticuerpos antifosfolipidos[4] si bien es interesante su aparición en algunos grupos de pacientes COVID-19 no se han relacionado directamente con eventos trombóticos y estaría a la prueba de investigaciones futuras de causalidad. En correlación a los estudios antes mencionados se evidencian serios trastornos desde el punto de vista de la hemostasia, la presencia de coagulación intravascular diseminada CID se ha descrito en un porcentaje considerable de hasta en el $71,4 \%$ de los pacientes no sobrevivientes[2], pero esta entidad clínica en el curso de la enfermedad por COVID-19 presenta un patrón de coagulopatía protrombótica diferente al reportado en la sepsis, donde el recuento de plaquetas, generalmente, disminuye y el tiempo de protrombina se prolonga con la tendencia hemorrágica asociada[33]. Estos datos son respaldados por una serie de 24 pacientes ingresados en la unidad de cuidados intensivos debido a COVID-19 quienes se le evaluaron de manera seriada con tromboelastografía los estados hemostáticos durante su estancia y en quienes cuyos resultados apoyan la hipercoagulabilidad junto con un estado inflamatorio severo[34].

Existen otros factores que interfieren en la severidad de la enfermedad por COVID-19 y son factores pronósticos en ésta, además, de estar asociados a mortalidad fuera de la esfera de la coagulación, como lo son los reactantes de fase aguda, distintivos actual de la patogénesis del SARS-CoV-2 que nos terminan de orientar sobre la respuesta inflamatoria del huésped y transpolar tales resultados hacia los efectos directos sobre la hemostasia y la gravedad de la endoteliopatía viral a causa de la desrregulación o alteración del estado de inmunotrombosis. Zeng et al.[35] describen en probablemente uno de los primeros metaanálisis que asocia marcadores inflamatorios con la severidad de la enfermedad por COVID-19, con un total de 3.962 pacientes incluidos en el análisis, reportaron que los resultados de efectos aleatorios demostraron que los pacientes con COVID-19 en el grupo no severo tenían niveles más bajos de PCR (DMP = $-41,78 \mathrm{mg} / \mathrm{l}, \mathrm{IC} 95 \%=[-52,43,-31,13], \mathrm{P}<0,001)$, PCT (DMP $=-0,13 \mathrm{ng} / \mathrm{ml}, \mathrm{IC} 95 \%=[-0,20,-0,05], \mathrm{P}$ $<0,001)$, IL-6 (DMP $=-21,32 \mathrm{ng} / \mathrm{l}, \mathrm{IC} 95 \%=[-28,34$, $-14,31], P<0,001), E S R(D M P=-8 \mathrm{~mm} / \mathrm{h}, \mathrm{IC} 95 \%$ $=[-14,-2], P=0,005)$, SAA (DMP $=-43,35 \mu \mathrm{g} / \mathrm{ml}, \mathrm{IC}$ $95 \%=[-80,85,-5,85], P=0,020)$ y ferritina sérica $(D M P=-398.80 \mathrm{mg} / \mathrm{l}, \mathrm{IC} 95 \%=[-625,89,-171,71]$, $\mathrm{P}<0,001$ ), en comparación con aquellos en el grupo severo. Además, los sobrevivientes tenían un nivel más bajo de IL-6 que los no sobrevivientes (DMP = $-4,80 \mathrm{ng} / \mathrm{ml}$, IC $95 \%=[-5,87,-3,73], \mathrm{P}<0,001)[35]$.
Entre otros factores de riesgo independientes que contribuyen a medir la gravedad de COVID-19 se encuentra el lactato deshidrogenasa LDH quien se ha reportado con valor de diagnóstico de severidad independiente una con sensibilidad máximas $(100,00 \%)$ y especificidad $(86,67 \%)$, con un valor de corte de 283 U/L[36]. Brandon et al.[37] evaluaron en un análisis agrupado de siete estudios los valores de LDH en un total de 1.206 pacientes incluidos con valores máximos de corte de 245 a 253,2 U/L encontrando que los niveles elevados de LDH se asociaron con un aumento de $>6$ veces en las probabilidades de desarrollar enfermedad grave y un aumento de $>16$ veces en las probabilidades de mortalidad en pacientes con COVID-19. La linfocitopenia es otra de las características destacadas de los pacientes críticos con COVID-19. Wang et al.[38] reportaron en 344 pacientes admitidos en $\mathrm{UCl}$ que ésta estaba presente en el $(69,5 \%)$ sujetos y fue predominante en los no sobrevivientes (91,6\% vs 55,7\%; P < 0,001); el recuento de linfocitos más alto se asoció significativamente con una disminución de la mortalidad en aquellos que presentaron un recuento $1,0 \times 10 \times 10$ 9/L a $1,0(\mathrm{HR}, 0,1$; IC del $95 \%, 0,06-0,18 ; \mathrm{P}<0,001)$.

\section{Evaluación del riesgo trombótico en el paciente quirúrgico COVID-19}

La evaluación del riesgo trombótico en cuanto al paciente quirúrgico COVID-19 para el momento de la revisión no existen consideraciones al respecto, sin embargo, los sistemas de puntuación que calculan el riesgo de TEV postoperatorio para pacientes individuales como la puntuación de Caprini[20], la cual ha sido validada para los procedimientos quirúrgicos en el estudio de validación de Bahl et al.[39] y ajustados por la profilaxis recibida, es hasta ahora uno de los sistemas más completos para evaluar el riesgo trombótico en el paciente quirúrgico no COVID-19 al tratarse de un sistema de puntuación simple y autónomo en la estratificación del riesgo quirúrgicos y la presencia de factores de riesgo especificados de trombosis previamente definidos, el riesgo de TEV se clasifica como muy bajo (0-1 punto), bajo ( 2 puntos), moderado (3-4 puntos) o alto ( $\geq 5$ puntos) los riesgos iniciales estimados de TEV fueron $<0,5 \%, 1,5 \%, 3,0 \%$ y $6,0 \%$ en pacientes con muy bajo, bajo, moderado y alto riesgo de TEV, respectivamente (después de ajustar por la profilaxis recibida)[39]. Los anteriores predictores y factores de riesgo de trombosis para la enfermedad de COVID-19 se han adaptado ajustados a las categorías presentes en la escala de Caprini en orden creciente de complejidad y en relación a la morbimor- 
talidad (Tabla 1). En este sentido la elección de la o las medidas que requiere cada paciente dependerá de su riesgo particular de desarrollar enfermedad tromboembólica venosas TEV el cual está en función del riesgo individual predicho de complicaciones trombóticas evaluado por la escala de Caprini contra el riesgo anticipado de complicaciones hemorrágicas quirúrgicas de la cirugía a la que será sometido. Con respecto a las medidas a tomar según las dosis de heparina, resumimos la recomendación actual.

En base a los estudios previos de tromboprofilaxis extendidas en los pacientes quirúrgicos no COVID-19 y su impacto en la disminución de la mortalidad y de eventos tromboembólicos, especialmente en pacientes de alto riesgo[40],[41],[42], los hallazgos recientes de las autopsias y los datos sobre el momento del diagnóstico de TEV en relación con la hospitalización sugieren claramente que los eventos tromboembólicos también contribuyen a morbilidad y mortalidad en el entorno ambulatorio en el paciente COVID-19[43], por lo que se propone el siguiente manejo en base las recomendaciones actuales.

\section{Riesgo estimado de sangrado}

La estratificación del riesgo de hemorragia tras el

\begin{tabular}{|c|c|c|c|}
\hline 1 Punto & 2 Puntos & 3 Puntos & 5 Puntos \\
\hline - Edad 41 a 60 años & - Edad de 61 a 74 años & - Edad mayor a 75 años & - ACV en el último mes \\
\hline - Cirugía menor & - Cirugía artroscópica & $\begin{array}{l}\text { - Antecedentes de embolia } \\
\text { venosa }\end{array}$ & - Artroplastia electiva \\
\hline - IMC > 25 kg/m² & $\begin{array}{l}\text { - Cirugía abierta mayor de } \\
45 \text { min }\end{array}$ & $\begin{array}{l}\text { - AP familiares de embolia } \\
\text { venosa }\end{array}$ & $\begin{array}{l}\text { - Fractura de cadera pelvis } \\
\text { o pierna }\end{array}$ \\
\hline - Edema en miembros & $\begin{array}{l}\text { - Cirugía laparoscópica } \\
\text { mayor de } 45 \text { min }\end{array}$ & - Factor V de Leiden & $\begin{array}{l}\text { - Lesión espinal aguda en el } \\
\text { último mes }\end{array}$ \\
\hline - Venas varicosas & - Cáncer & - Protrombina 20210A & - Paciente en UCI \\
\hline - Embarazo o puerperio & $\begin{array}{l}\text { - Paciente encamado por } \\
\text { más de } 72 \text { h }\end{array}$ & - Anticoagulante lúpico & - Dímero-D > 3.000 ng/m \\
\hline $\begin{array}{l}\text { - Historia de abortos espon- } \\
\text { táneos }\end{array}$ & - Paciente inmovilizado & $\begin{array}{l}\text { - Anticuerpos anticardio- } \\
\text { lipina }\end{array}$ & $\begin{array}{l}\text { - Linfocitopenia < } 800 \\
x 10^{\wedge} 6 / L\end{array}$ \\
\hline - $\mathrm{ACO}$ & - Acceso venoso central & $\begin{array}{l}\text { - Niveles séricos homocístei- } \\
\text { na elevados }\end{array}$ & $\begin{array}{l}\text { - Plaquetas entre } 0-50 \times \\
10^{9} / L\end{array}$ \\
\hline - Sepsis en el último mes & & $\begin{array}{l}\text { - Trombocitopenia inducida } \\
\text { por heparina }\end{array}$ & $\begin{array}{l}\text { - Lactato deshidrogenasa } \\
\mathrm{LDH}>283 \mathrm{U} / \mathrm{L}\end{array}$ \\
\hline $\begin{array}{l}\text { - Neumonía en el último } \\
\text { mes o EPOC }\end{array}$ & & $\begin{array}{l}\text { - Trombofilia congénita o } \\
\text { adquirida }\end{array}$ & \\
\hline $\begin{array}{l}\text { - Pruebas función pulmonar } \\
\text { anormales }\end{array}$ & & $\begin{array}{l}\text { - Proteína C reactiva }(P C R)> \\
150 \mathrm{mg} / \mathrm{L}\end{array}$ & \\
\hline - Infarto cardiaco & & - Dímero D >1.500 ng/mL & \\
\hline $\begin{array}{l}\text { - Enfermedad inflamatoria } \\
\text { Intestinal }\end{array}$ & & - Ferritina $>1.000 \mathrm{ng} / \mathrm{mL}$ & \\
\hline - Encamamiento & & $\begin{array}{l}\cdot \text { Linfocitopenia > } 1.0 x \\
10^{\wedge} 6 / L\end{array}$ & \\
\hline \multirow[t]{3}{*}{ - Sexo masculino } & & - IL-6 > $40 \mathrm{pg} / \mathrm{mL}$ & \\
\hline & & $\begin{array}{l}\text { - Plaquetas entre } 50-100 \\
\times 10^{9} / \mathrm{L}\end{array}$ & \\
\hline & & $\begin{array}{l}\text { - Lactato deshidrogenasa } \\
\text { LDH } 245 \text { a } 283 \mathrm{U} / \mathrm{L}\end{array}$ & \\
\hline - COVID-19 asintomático & $\begin{array}{l}\text { - COVID-19 enfermedad } \\
\text { leve/ moderada estable }\end{array}$ & $\begin{array}{l}\text { - COVID-19 enfermedad } \\
\text { grave o }\end{array}$ & - COVID-19 crítico \\
\hline
\end{tabular}


uso de tromboprofilaxis postoperatoria es un apartado aún no lúcido, en ausencia de datos de estudios observacionales amplios, prospectivos y basados en la población quirúrgica, el riesgo basal de hemorragia puede derivarse de los grupos de control (placebo o sin profilaxis farmacológica) en ensayos aleatorios. Es limitada la investigación que ha intentado identificar factores de riesgo para hemorragia, aunque algunos estudios han identificado factores de riesgo en pacientes sometidos a cirugía de cáncer gástrico, pancreaticoduodenectomía, resección hepática parcial y cirugía abdominal mixta, los cuales son de gran ayuda al momento de evaluar el riesgo de sangrado (Tabla 2). Sin embargo, el riesgo de hemorragia mayor tras procedimientos quirúrgicos mayores ha sido estimado en 1,2\% (IC 95\%, 0,9\%-1,7\%), mientras que el hematoma y hemorragia de la herida que requería reoperación es de 0,8\% y 0,7\% respectivamente[44].

\section{Discusión}

Es de recordar que el órgano más grande y multifuncional del organismo como lo es el endotelio vascular es un alto expreso de los receptores ACE2, la ruptura del equilibrio hemostático generado por el SARS-CoV-2 debido a una respuesta inflamatoria significativa y abrumadora hacia el huésped y la endotelitis viral[55] generada con daño estructural del glicocalix es en síntesis el estado de falla sanguínea[54] observado en estos pacientes con características únicas, explica en cierto grado el aumento exponencial de la enfermedad tromboembólica y a la vez explica la alta frecuencia de TEV particularmente en pacientes con mayor estado de gravedad e ingresados en la UCI.

En cuanto al riesgo tromboembólico en el paciente no quirúrgico COVID-19 la TEV es de $20 \%$, pero con incidencias acumulativas de hasta el $49 \%$ durante la hospitalización[23], especialmente en los más graves, cuando se comparan estas cifras con los pacientes quirúrgicos hospitalizados por cirugía mayor en no COVID-19 a pesar de las terapias tromboprofilácticas preestablecidas, el radio estadístico es muy similar rondando el 15\%-40\% y llegando a representar hasta el $60 \%$ en cirugías ortopédicas[44], sin embargo, no se ha establecido la relación de esta patología con la probable sumatoria de efectos tromboembólicos generados a partir de la injuria quirúrgica.

Los estudios y las nuevas investigaciones en cuanto a relación entre los procedimientos quirúrgicos y los pacientes COVID-19 diagnosticados en el perioperatorio han revelado el aumento de desenlaces fatales en este grupo específico de pacientes con au- mento de las complicaciones postoperatorias. En un estudio multicéntrico internacional con la participación de 24 países donde se evaluaron procedimientos electivos y de emergencia, reportó una mortalidad global desproporcionadamente alta a los 30 días de $23,8 \%$ en todos los subgrupos. El $81,7 \%$ de los fallecidos experimentaron complicaciones pulmonares, sin definir qué número de pacientes pudieron cursar con cuadros enmascarados de EP en conjunto con los cuadros inestabilidad hemodinámica e hipoxemia, aunque se reporta $2 \%$ de EP global, a pesar de que no se pudieron seguir un porcentaje similar de pacientes con esta complicación por falta de datos. Esta tasa de mortalidad postoperatoria es muy semejante a las reportadas en los pacientes médicos más críticos ingresados en UCI con COVID-19[45]. Por lo tanto, los pacientes que contraen la infección por SARSCoV-2 y son sometidos a intervenciones quirúrgicas, independientemente de su nivel de complejidad, son pacientes catalogados con riesgo per se, es por ello que a la adaptación de la escala de Caprini se realiza una reestructuración de los riesgos de clasificación anterior, dejando por fuera al acápite muy bajo riesgo ya que ante las justificaciones anteriores en nuestra investigación ningún paciente presentó muy bajos riesgos de complicaciones, si a esto se le suma la injuria quirúrgica a pesar de ser un campo no bien dilucidado para el momento.

Debido al estado procoagulante de esta enfermedad las guías e instituciones internacionales recomiendan la profilaxis farmacológica de TVP en todos los pacientes hospitalizados[46],[47],[48],[49]. Sin embargo, estas recomendaciones se basan en la tromboprofilaxis empíricas generales y no son específicas para pacientes COVID-19, menos aún en el subgrupo quirúrgico de esta patología, aunado a esto, no existe un acuerdo general sobre la dosificación óptima en este contexto, no obstante, los beneficios son vistos especialmente en pacientes graves con disminución notable de la mortalidad con el uso de heparina versus usuarios sin heparina (32,8\% vs 52,4\%, $\mathrm{p}=0,017)$ incluso en los pacientes con criterios de coagulación intravascular diseminada CID (40,0\% vs $64,2 \%, p=0,029)[50]$. La consideración de dosis aumentadas para los pacientes más críticos con dosis terapéuticas, están justificadas según los reportes FA Klok et al.[8] y Llitjos[51] por separados, evidenciaron que los pacientes adultos críticos con COVID-19 pueden desarrollar TEV con la profilaxis farmacológica estándar. El estudio de Paranjpe et al.[52] en Nueva York que incluyó a 2.773 pacientes hospitalizados, de los cuales 786 (23\%) estaban en tratamiento a dosis terapéuticas de heparina de bajo peso molecular 


\section{Tabla 2. Factores que aumentan el riesgo de hemorragia en pacientes quirúrgicos}

\begin{tabular}{|c|c|}
\hline Factores de riesgo general & Factores dependientes del procedimiento/ intervención \\
\hline - Sangrado activo & $\begin{array}{l}\text { *Cirugía abdominal } \\
\text { - Varón } \\
\text { - Hemoglobina }<13 \mathrm{~g} / \mathrm{dl} \text {, } \\
\text { - Cáncer } \\
\text { - cirugía complicada definido por dos o más procedimientos } \\
\text { - Disección difícil o más de una anastomosis }\end{array}$ \\
\hline - Antecedentes de sangrado grave & $\begin{array}{l}\text { *Pancreatoduodenectomía } \\
\text { - Sepsis, fuga pancreática o sangrado centinela }\end{array}$ \\
\hline $\begin{array}{l}\text { - Trastornos de la coagulación conoci- } \\
\text { dos, no tratados }\end{array}$ & $\begin{array}{l}\text { *Resección hepática } \\
\text { Número de segmentos, resección extrahepática concomitante, hepatocarcinoma, } \\
\text { anemia y plaquetopenia }\end{array}$ \\
\hline - Insuficiencia hepática o renal graves & $\begin{array}{l}\text { * Cirugía cardiaca } \\
\text { - Uso de AAS } \\
\text { - Uso de clopidogrel en los } 3 \text { días previos a la cirugía } \\
\text { - IMC }>25 \mathrm{~kg} / \mathrm{m}^{2} \text {, cirugía urgente, colocación de } 5 \text { o más bypass } \\
\text { - Edad avanzada, insuficiencia renal, cirugía distinta a la revascularización, mayor } \\
\text { duración de la circulación extracorpórea }\end{array}$ \\
\hline - Trombopenia & $\begin{array}{l}\text { * Cirugía torácica } \\
\text { Neumonectomia o resección extendida }\end{array}$ \\
\hline - Ictus agudo & $\begin{array}{l}\text { Procedimientos en los que las complicaciones hemorrágicas pueden tener conse- } \\
\text { cuencias graves: } \\
\text { - Cirugía ocular } \\
\text { - Craneotomía } \\
\text { - Cirugía espinal } \\
\text { - Trauma espinal } \\
\text { - Procedimientos reconstructivos con colgajo libre }\end{array}$ \\
\hline \multicolumn{2}{|l|}{ - Hipertensión arterial no controlada } \\
\hline \multicolumn{2}{|l|}{$\begin{array}{l}\text { - Punción lumbar, anestesia espinal o } \\
\text { epidural en las primeras } 12 \mathrm{~h} \text { tras la } \\
\text { administración de HBPM o si se admi- } \\
\text { nistra la HBPM en las primeras } 6 \text { h tras } \\
\text { la técnica }\end{array}$} \\
\hline $\begin{array}{l}\text { - Uso concomitante de anticoagulan- } \\
\text { tes, antiplaquetarios o trombolíticos }\end{array}$ & \\
\hline
\end{tabular}

IMC: Índice de masa corporal; AAS: Ácido acetilsalicílico.

Tomado de: Michael K. Gould et al. Prevention of VTE in Nonorthopedic Surgical PatientsAntithrombotic Therapy and Prevention of Thrombosis, 9th ed: American College of Chest Physicians Evidence-Based Clinical Practice Guidelines. 2012.

HBPM, demostró de manera interesante una mortalidad hospitalaria del $29,1 \%$ con una mediana de supervivencia de 21 días para aquellos que recibieron dosis terapéuticas, en comparación con $62,7 \%$ con una mediana de supervivencia de 9 días para aquellos que no recibieron, resultados similares y una mejora en los parámetros ventilatorios y gasométricos se encontraron en el ensayo clínico aleatorizado de fase II HESACOVID[56].

La utilización de terapias anticoagulante en el paciente crítico quirúrgico COVID-19, probablemente sea el tema de mayor complejidad en cuanto a ajustar las pautas en pro de la disminución de los eventos tromboembólicos por el alto riesgo de sangrado, por lo que los estudios futuros estarán comprometidos en qué podemos mejorar y hacia dónde extender la terapia tromboprofiláctica en este grupo de pacientes. Ante la falta de evidencia en este entorno, se recomienda guiar las terapias tromboprofilácticas en el paciente quirúrgico COVID-19 de manera individual con orientación y apoyo de las pautas de la Sociedad Estadounidense de Hematología ASH[53], las cuales 
destacan por su alcance, que incluye cuestiones generales relevantes para cualquier procedimiento quirúrgico y aquellas relacionadas con subespecialidades quirúrgicas. No obstante, en una revisión reciente de las recomendaciones y pautas para el manejo de la coagulopatía y trombosis en pacientes críticamente enfermos con COVID-19 de diferentes sociedades se pueden encontrar similitudes de manejo, descritas anteriormente, pero sin ningún consenso al respecto por la falta de ensayos clínicos aleatorizados que justifiquen las recomendaciones y, en ausencia de estas adoptan un manejo individualizado en este tipo de pacientes[57].

\section{Conclusiones}

Las complicaciones tromboembólicas representan una evidente causa de morbimortalidad en el pacien- te COVID-19 por la gran afectación del endotelio vascular generado por el SARS-CoV-2, la relación y causalidad de esta patología con el aumento de eventos tromboembólicos como se podría esperar al sumar la injuria quirúrgica con la respuesta inflamatoria sistémica es aún un campo por explorar. Identificar a los pacientes susceptibles de desarrollar complicaciones y al unísono de trombosis a través del sistema de puntuación modificado de Caprini para esta emergente patología es la base para guiar y establecer estrategias óptimas de tromboprofilaxis, ya sea con dosis estándar, aumentadas o terapéuticas ajustadas siempre al riesgo conocido de hemorragia en el contexto postoperatorio. El llamado es a realizar ensayos clínicos prospectivos aleatorizados para abordar los problemas de anticoagulación en este grupo específico de pacientes, además, de proporcionar mayores datos en cuanto al impacto de la cirugía y el estado de la enfermedad de la COVID-19.

\section{Referencias}

1. Li Q, Guan X, Wu P, Wang X, Zhou L, Tong $Y$, et al. Early transmission dynamics in Wuhan, China, of Novel Coronavirusinfected pneumonia. N Engl J Med. 2020 Mar;382(13):1199207. https://doi.org/10.1056/NEJMoa2001316 PMID:31995857

2. Tang $N$, Li D, Wang $X$, Sun Z. Abnormal coagulation parameters are associated with poor prognosis in patients with novel coronavirus pneumonia. J Thromb Haemost. 2020 Apr;18(4):844-7. https:// doi.org/10.1111/jth.14768 PMID:32073213

3. Liu Z, Xiao X, Wei X, Li J, Yang J, Tan $\mathrm{H}$, et al. Composition and divergence of coronavirus spike proteins and host ACE2 receptors predict potential intermediate hosts of SARS-CoV-2. J Med Virol. 2020 Jun;92(6):595601. https://doi.org/10.1002/ jmv.25726 PMID:32100877

4. Debuc B, Smadja DM. Is COVID-19 a New Hematologic
Disease? Stem Cell Rev Rep. 2020 May;1-5: https://doi. org/10.1007/s12015-02009987-4 PMID:32399806

5. Iba T, Levy JH, Levi M, Connors JM, Thachil J. Coagulopathy of Coronavirus Disease 2019. Crit Care Med. 2020 Sep;48(9):135864. https://doi.org/10.1097/ CCM.0000000000004458 PMID:32467443

6. Varga Z, Flammer AJ, Steiger $P$, Haberecker M, Andermatt $R$, Zinkernagel AS, et al. Endothelial cell infection and endotheliitis in COVID-19. Lancet. 2020 May;395(10234):14178. https://doi.org/10.1016/ S0140-6736(20)30937-5 PMID:32325026

7. Cui S, Chen S, Li X, Liu S, Wang $F$. Prevalence of venous thromboembolism in patients with severe novel coronavirus pneumonia. J Thromb Haemost. 2020 Jun;18(6):1421-4. https:// doi.org/10.1111/jth.14830 PMID:32271988

8. Klok FA, Kruip MJ, van der Meer NJ, Arbous MS, Gom- mers DA, Kant KM, et al. Incidence of thrombotic complications in critically ill ICU patients with COVID-19. Thromb Res. 2020 Jul;191:1457. https://doi.org/10.1016/j. thromres.2020.04.013 PMID:32291094

9. Chen J, Wang $X$, Zhang $S$, et al. (2020) Findings of acute pulmonary embolism in COVID-19 patients (3/1/2020). SSRN. https:// doi.org/10.2139/ssrn.3548771.

10. Daniel E. Leisman, Clifford S. Deutschman, Matthieu Legrand. Facing COVID-19 in the ICU: vascular dysfunction, thrombosis, and dysregulated inflammation. Intensive Care Med. 2020 Apr;1-4(6):1105-8. https:// doi.org/10.1007/s00134-02006059-6.

11. Coccolini F, Perrone G, Chiarugi M, Di Marzo F, Ansaloni L, Scandroglio I, et al. Surgery in COVID-19 patients: operational directives. World J Emerg Surg. 2020 Apr;15(1):25. https:// doi.org/10.1186/s13017-02000307-2 PMID:32264898 
12. SAGES and EAES Recommendations Regarding Surgical Response to COVID-19 Crisis - SAGES [Internet]. Available from: https:// www.sages.org/recommendations-surgical-response-covid-19 [consultada el 5 de junio de 2020].

13. Besnier E, Tuech JJ, Schwarz L. We Asked the Experts: Covid-19 Outbreak: Is There Still a Place for Scheduled Surgery? "Reflection from Pathophysiological Data". World J Surg. 2020 Jun;44(6):1695-8. https:// doi.org/10.1007/s00268-02005501-6 PMID:32246185

14. Tao KX, Zhang BX, Zhang $P$, Zhu $P$, Wang GB, Chen XP; General Surgery Branch of Hubei Medical Association, General Surgery Branch of Wuhan Medical Association. [Recommendations for general surgery clinical practice in 2019 coronavirus disease situation]. Zhonghua Wai Ke Za Zhi. 2020 Mar;58(3):170-7. PMID:32187920

15. Aminian A, Safari S, RazeghianJahromi A, Ghorbani M, Delaney CP. COVID-19 Outbreak and Surgical Practice: Unexpected Fatality in Perioperative Period. Ann Surg. 2020 Jul;272(1):e27-9. https://doi.org/10.1097/ SLA.0000000000003925 PMID:32221117

16. Lei $S$, Jiang F, Su W, Chen C, Chen J, Mei W, et al. Clinical characteristics and outcomes of patients undergoing surgeries during the incubation period of COVID-19 infection. EClinicalMedicine. 2020 Apr;21:100331. https://doi.org/10.1016/j. eclinm.2020.100331 PMID:32292899

17. Parohan M, Yaghoubi S, Seraji A, Javanbakht MH, et al. Risk factors for mortality in patients with Coronavirus disease 2019 (COVID-19) infection: a systematic review and meta-analysis of observational studies. medRxiv preprint https://doi.org/10.1101/ 2020.04.09.20056291.

18. Thachil J, Tang N, Gando S, Falanga A, Cattaneo M, Levi M, et al. ISTH interim guidance on recognition and management of coagulopathy in COVID-19. J Thromb Haemost. 2020 May;18(5):1023-6. https:// doi.org/10.1111/jth. 14810 PMID:32338827

19. Anderson DR, Morgano GP, Bennett C, Dentali F, Francis CW, Garcia DA, et al. American Society of Hematology 2019 guidelines for management of venous thromboembolism: prevention of venous thromboembolism in surgical hospitalized patients. Blood Adv. 2019 Dec;3(23):3898-

944. https://doi.org/10.1182/ bloodadvances.2019000975 PMID:31794602

20. Joseph A. Caprini, Jzldn I. Arcehs, and Jose'J. Reyna. Effective Risk Stratification of Surgical and Nonsurgical Patients for Venous Thromboembolic Disease. Seminars in Hematology, Vol38, NO 2, Suppl5 (April), 2001: PP 1219.

21. Di Micco P, Russo V, Carannante $\mathrm{N}$, Imparato M, Rodolfi S, Cardillo $\mathrm{G}$, et al. Clotting Factors in COVID-19: Epidemiological Association and Prognostic Values in Different Clinical Presentations in an Italian Cohort. J Clin Med. 2020 May;9(5):1371. https:// doi.org/10.3390/jcm9051371 PMID:32392741

22. Tang $N$, Li D, Wang $X$, Sun Z. Abnormal coagulation parameters are associated with poor prognosis in patients with novel coronavirus pneumonia. J Thromb Haemost. 2020 Apr;18(4):844-7. https:// doi.org/10.1111/jth.14768 PMID:32073213

23. Al-Ani F, Chehade S, LazoLangner A. Thrombosis risk associated with COVID-19 infection. A scoping review. Thromb Res. 2020 Aug;192:15260. https://doi.org/10.1016/j. thromres.2020.05.039 PMID:32485418

24. Liang W, Liang $\mathrm{H}, \mathrm{Ou} L$, Chen $B$, Chen A, Li C, et al.; China Medical Treatment Expert Group for COVID-19. Development and Validation of a Clinical Risk Score to Predict the Occurrence of Critical Illness in Hospitalized Patients With COVID-19. JAMA Intern Med. 2020 Aug;180(8):10819. https://doi.org/10.1001/ jamainternmed.2020.2033 PMID:32396163

25. Tian $\mathbf{W}$, Jiang $\mathbf{W}$, Yao J, Nicholson CJ, Li RH, Sigurslid HH, et al. Predictors of mortality in hospitalized COVID-19 patients: A systematic review and meta-analysis. J Med Virol. 2020 May;92(10):1875-83. https:// doi.org/10.1002/jmv.26050 PMID:32441789

26. Lippi G, Favaloro EJ. D-dimer is associated with severity of coronavirus disease 2019: a pooled analysis. Thromb Haemost. 2020 May;120(5):876-8. https://doi. org/10.1055/s-0040-1709650 PMID:32246450

27. Guan WJ, Ni ZY, Hu Y, Liang WH, Ou CQ, He JX, et al.; China Medical Treatment Expert Group for Covid-19. Clinical characteristics of coronavirus disease 2019 in China. N Engl J Med. 2020 Apr;382(18):1708-20. https://doi.org/10.1056/NEJMoa2002032 PMID:32109013

28. Wang D, Hu B, Hu C, Zhu F, Liu $X$, Zhang J, et al. Clinical characteristics of 138 hospitalized patients with 2019 novel coronavirus-infected pneumonia in Wuhan, China. JAMA. 2020 Mar;323(11):1061-9. https://doi. org/10.1001/jama.2020.1585 PMID:32031570

29. Huang C, Wang Y, Li X. Clinical 
features of patients infected with 2019 novel coronavirus in Wuhan, China. Lancet (Lond. Engl.) 2020;395:497-506.

30. Ranucci M, Ballotta A, Di Dedda U, Bayshnikova E, Dei Poli M, Resta $\mathrm{M}$, et al. The procoagulant pattern of patients with $\mathrm{CO}$ VID-19 acute respiratory distress syndrome. J Thromb Haemost. 2020 Jul;18(7):1747-51. https:// doi.org/10.1111/jth.14854 PMID:32302448

31. Zhou F., Yu T., Du R. Clinical course and risk factors for mortality of adult inpatients with COVID-19 in Wuhan, China: a retrospective cohort study. Lancet (Lond. Engl.) 2020;395:10541062.

32. Yang $X$, Yang $Q$, Wang $Y$, Wu $Y, X u$ J, Yu Y, et al. Thrombocytopenia and its association with mortality in patients with COVID-19. J Thromb Haemost. 2020 Jun;18(6):1469-72. https:// doi.org/10.1111/jth.14848 PMID:32302435

33. Ranucci M, Ballotta A, Di Dedda U, Bayshnikova E, Dei Poli M, Resta $\mathrm{M}$, et al. The procoagulant pattern of patients with $\mathrm{CO}$ VID-19 acute respiratory distress syndrome. J Thromb Haemost. 2020 Jul;18(7):1747-51. https:// doi.org/10.1111/jth.14854 PMID:32302448

34. Panigada M, Bottino N, Tagliabue P, Grasselli G, Novembrino C, Chantarangkul V, et al. Hypercoagulability of COVID-19 patients in intensive care unit: A report of thromboelastography findings and other parameters of hemostasis. J Thromb Haemost. 2020 Jul;18(7):1738-42. https:// doi.org/10.1111/jth.14850 PMID:32302438

35. Urong Zeng, Yuzhao Huang, Ying Guo, Xiang Chen, Liang Xiao, Guangtong Deng. Association of inflammatory markers with the severity of COVID-19:
A meta-analysis. https://doi. org/10.1016/j.ijid.2020.05.055.

36. Han $Y$, Zhang H, Mu S, et al. Lactate dehydrogenase, a Risk Factor of Severe COVID-19 Patients. medRxiv preprint 2020. https://doi.org/10.1101/2020.03. 24.20040162.

37. Henry BM, Aggarwal G, Wong J, Benoit S, Vikse J, Plebani M, et al.; Brandon Michael Henry. Gaurav Aggarwal,b Johnny Wong. Lactate dehydrogenase levels predict coronavirus disease 2019 (COVID-19) severity and mortality: A pooled analysis. Am J Emerg Med. 2020 May;38(9):17226. https://doi.org/10.1016/j. ajem.2020.05.073.

38. Wang Y, Lu X, Li Y, Chen H, Chen $T$, Su N, et al. Clinical Course and Outcomes of 344 Intensive Care Patients with COVID-19. Am J Respir Crit Care Med. 2020 Jun;201(11):14304. https://doi.org/10.1164/ rccm.202003-0736LE PMID:32267160

39. Bahl V, Hu HM, Henke PK, Wakefield TW, Campbell DA Jr, Caprini JA. A validation study of a retrospective venous thromboembolism risk scoring method. Ann Surg. 2010 Feb;251(2):34450. https://doi.org/10.1097/ SLA.0b013e3181b7fca6 PMID:19779324

40. Huo MH, Muntz J. Extended thromboprophylaxis with lowmolecular-weight heparins after hospital discharge in high-risk surgical and medical patients: a review. Clin Ther. 2009 Jun;31(6):1129-41. https:// doi.org/10.1016/j.clinthera.2009.06.002 PMID:19695383

41. Bottaro FJ, Elizondo MC, Doti C, Bruetman JE, Perez Moreno PD, Bullorsky EO, et al. Efficacy of extended thrombo-prophylaxis in major abdominal surgery: what does the evidence show? A meta-analysis. Thromb Haemost. 2008 Jun;99(6):1104-11. https:// doi.org/10.1160/TH07-12-0759 PMID:18521515

42. Felder $S$, Rasmussen MS, King R, Sklow B, Kwaan M, Madoff $\mathrm{R}$, et al. Prolonged thromboprophylaxis with low molecular weight heparin for abdominal or pelvic surgery. Cochrane Database Syst Rev. 2019 Mar;3(3):CD004318. https:// doi.org/10.1002/14651858. CD004318.pub4 PMID:30916777

43. Langer $F$, Kluge $S$, Klamroth $\mathrm{R}$, Oldenburg J. Florian Langer 1, Stefan Kluge 2, Robert Klamroth 3, Johannes Oldenburg. Coagulopathy in COVID-19 and Its Implication for Safe and Efficacious Thromboprophylaxis [Online ahead of print.]. Hamostaseologie. 2020 Jun;40(03):264-9. https://doi. org/10.1055/a-1178-3551.

44. Michael K. Gould, David A. Garcia, Sherry M. Wren et al. Prevention of VTE in Nonorthopedic Surgical Patients Antithrombotic Therapy and Prevention of Thrombosis, 9th ed: American College of Chest Physicians Evidence-Based Clinical Practice Guidelines. Chest. 2012 Feb;141(2 Suppl):e227S77S. https://doi.org/10.1378/ chest.11-2297 PMID:22315263

45. COVIDSurg Collaborative. Mortality and pulmonary complications in patients undergoing surgery with perioperative SARS-CoV-2 infection: an international cohort study. Published Online May 29, 2020 https://doi.org/10.1016/ S0140-6736(20)31182-X.

46. Thachil J, Tang N, Gando S, Falanga A, Cattaneo M, Levi M, et al. ISTH interim guidance on recognition and management of coagulopathy in COVID-19. J Thromb Haemost. 2020 May;18(5):1023-6. https:// 
doi.org/10.1111/jth. 14810

PMID:32338827

47. Bikdeli B, Madhavan MV, Jimenez D, Chuich T, Dreyfus I, Dri-

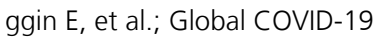
Thrombosis Collaborative Group, Endorsed by the ISTH, NATF, ESVM, and the IUA, Supported by the ESC Working Group on Pulmonary Circulation and Right Ventricular Function. COVID-19 and thrombotic or thromboembolic disease: implications for prevention, antithrombotic therapy, and followup. J Am Coll Cardiol. 2020 Jun;75(23):2950-73. https://doi. org/10.1016/j.jacc.2020.04.031 PMID:32311448

48. Barnes GD, Burnett A, Allen A, Blumenstein M, Clark NP, Cuker A, et al. Thromboembolism and anticoagulant therapy during the COVID-19 pandemic: interim clinical guidance from the anticoagulation forum. J Thromb Thrombolysis. 2020 Jul;50(1):72-81. https://doi. org/10.1007/s11239-02002138-z PMID:32440883

49. Recomendaciones de tromboprofilaxis y tratamiento antitrombótico en pacientes con COVID-19. Fecha de publicación: Actualizado: 29 de abril de 2020. Https://www.covid-19. seth.es/recomendaciones-detromboprofilaxis-y-tratamientoantitrombotico-en-pacientescon-covid-19/. Revisado en línea 3/5/2020.
50. Tang N, Bai H, Chen X, Gong J, Li D, Sun Z. Anticoagulant treatment is associated with decreased mortality in severe coronavirus disease 2019 patients with coagulopathy. J Thromb Haemost. 2020 May;18(5):10949. https://doi.org/10.1111/ jth.14817 PMID:32220112

51. Llitjos JF, Leclerc M, Chochois C, Monsallier JM, Ramakers M, Auvray $M$, et al. High incidence of venous thromboembolic events in anticoagulated severe COVID-19 patients. J Thromb Haemost. 2020 Jul;18(7):17436. https://doi.org/10.1111/ jth.14869 PMID:32320517

52. Paranjpe I, Fuster V, Lala A, Russak AJ, Glicksberg BS, Levin MA, et al. Association of Treatment Dose Anticoagulation With In-Hospital Survival Among Hospitalized Patients With COVID-19. J Am Coll Cardiol. 2020 Jul;76(1):122-4. https://doi. org/10.1016/j.jacc.2020.05.001 PMID:32387623

53. Anderson DR, Morgano GP, Bennett C, Dentali F, Francis CW, Garcia DA, et al. American Society of Hematology 2019 guidelines for management of venous thromboembolism: prevention of venous thromboembolism in surgical hospitalized patients. Blood Adv. 2019 Dec;3(23):3898-

944. https://doi.org/10.1182/ bloodadvances. 2019000975 PMID:31794602

54. Bjerkvig CK, Strandenes G, Elias- sen HS, Spinella PC, Fosse TK, Cap AP, et al. "Blood failure" time to view blood as an organ: how oxygen debt contributes to blood failure and its implications for remote damage control resuscitation. Transfusion. 2016 Apr;56 Suppl 2:S182-9. https:// doi.org/10.1111/trf.13500 PMID:27100755

55. Gu X, Zhou F, Wang Y, Fan G, Cao B. Respiratory viral sepsis: epidemiology, pathophysiology, diagnosis and treatment [https://doi. org/10.1183/16000617.00382020]. Eur Respir Rev. 2020 Jul;29(157):200038. https://doi. org/10.1183/16000617.00382020 PMID:32699026

56. Bertoldi A, Do Espírito Santo D, Salvetti M, et al. Therapeutic versus prophylactic anticoagulation for severe COVID-19: A randomized phase II clinical trial (HESACOVID). Thromb Res. 2020 Dec;196:359-66. https://doi.org/10.1016/j. thromres.2020.09.026.

57. Flaczyk A, Rosovsky RP, Reed CT, Bankhead-Kendall BK, Bittner EA, Chang MG. Comparison of published guidelines for management of coagulopathy and thrombosis in critically ill patients with COVID 19: implications for clinical practice and future investigations. Crit Care. 2020 Sep;24(1):559. https:// doi.org/10.1186/s13054-02003273-y PMID:32938471 